JOURNAL DE PHYSIQUE IV

Colloque C2, supplément au Journal de Physique II, Volume 5, février 1995

C2-129

\title{
Electron-Phonon Interaction and Phonon Softening in Systems with Magnetovolume Instabilities
}

\author{
H.C. Herper, P. Entel and W. Weber* \\ Theoretische Tieftemperaturphysik, Gerhard-Mercator-Universität, Gesamthochschule Duisburg, \\ 47048 Duisburg, Germany \\ * Theoretische Physik, Universität Dortmund, 44227 Dortmund, Germany
}

\begin{abstract}
The interrelation of Invar and martensitic behaviour in transition metal alloys like $\mathrm{Fe}_{65} \mathrm{Ni}_{35}$ is discussed on the basis of ab initio calculations for stoichiometric systems like $\mathrm{Fe}_{3} \mathrm{Ni}$. We also examined face centered cubic iron as a model system for Antiinvar which like Invar shows a pronounced phonon softening. Neutron scattering experiments on Invar systems have shown that the $\mathrm{TA}_{1}$ phonon mode softens for small wavevectors $\vec{q}$ along the [110] direction in the fcc lattice.

To day there exist only a few $a b$ initio calculations for martensitic transformations in nonmagnetic compounds. We have extended these kinds of investigations to the magnetic case. Our numerical calculations are based on the first principles theory of Varma and Weber who considered the electron-phonon interaction in second order perturbation theory. We have calculated renormalized phonon dispersion curves with the help of an appropriate set of tight-binding states fitted to $a b$ initio band structure results. Our numerical results reproduce the experimental findings. In particular we observe that phonon softening in systems with magnetovolume instabilities is caused by the long-range contribution to the electronphonon interaction.
\end{abstract}

\section{Introduction}

Neutron scattering experiments have shown that soft phonons exist in $\gamma$-iron and in a series of iron alloys [1,2]. We have examined the behaviour of pure iron and $\mathrm{Fe}_{3} \mathrm{Ni}$ with $\mathrm{Fe}_{3} \mathrm{Ni}$ as model system with similar properties as $\mathrm{Fe}_{65} \mathrm{Ni}_{35}$. Both systems undergo a martensitic $\gamma-\alpha$-phase transformation which is accompanied by onset of ferromagnetic order. The $\mathrm{Fe}_{3} \mathrm{Ni}$ system is of particular interest since it is close in concentration to the Invar region. On the other hand $\gamma$-iron is a famous Antiinvar system. So both are ideal systems for studying the connection between Invar, Antiinvar and the structural phase transformation.

In this paper we try to examine the nature of the phonon softening and to find out whether these vibrational effects are the precursor of the martensitic phase transition.

To this we have calculated the phonon dispersion by using the theory of Varma and Weber $[3,4]$ which is based on second order perturbation theory. In addition we have used the $a b$ initio augmented spherical wave (ASW) method in the framework of LDA to calculate the bandstructure of $\mathrm{Fe}_{3} \mathrm{Ni}$, fce iron and bcc iron. This method is a powerful tool and was first suggested by Williams, Kübler and Gelatt [5] and later dealt with by Morruzi [6], Entel et al. [7] and others. 


\section{Method}

In order to evaluate the electron-phonon matrix elements which are needed as input when making use of the procedure of Varma and Weber, the ASW band structure has been replaced by a SlaterKoster tight-binding scheme. This allows us to get information about the volume dependence of the electronic one particle states and of the transfer integral. The resulting tight-binding band structure with $2 * 36$ valence bands for the magnetic state $\left(6.4 \mu_{B}\right.$ per cell) is in good agreement with the ASW results and is shown in Fig. 1.
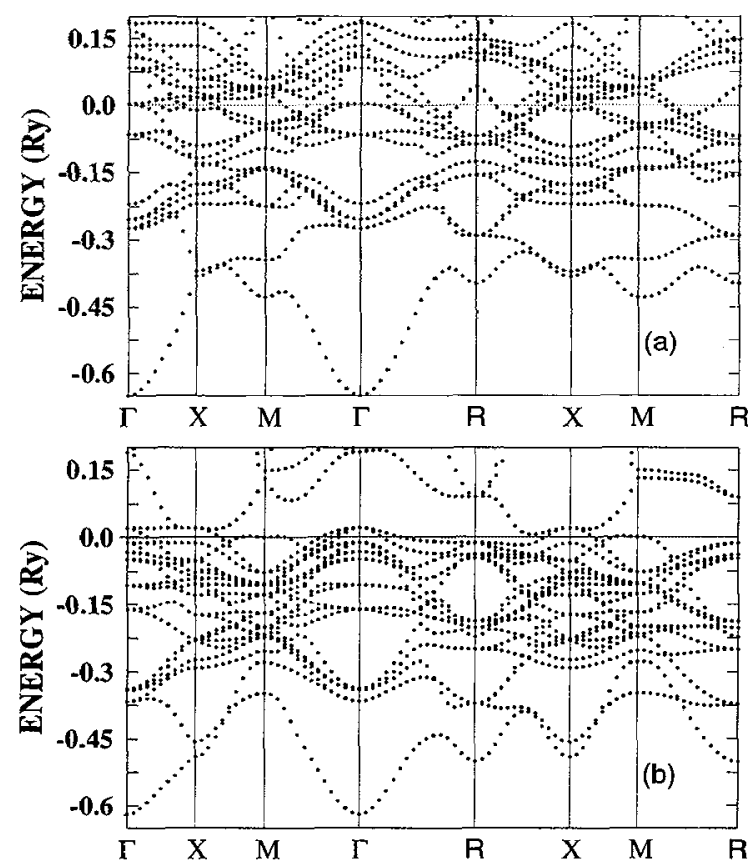

Figure 1: Minority-spin (a) and majority-spin (b) bands of fcc $\mathrm{Fe}_{3} \mathrm{Ni}$ as obtained from a tight-binding fit to ASW results. Bands are plotted along symmetry lines of the Brillouin zone of the simple cubic lattice. The magnetization is $6.4 \mu_{B}$ per cell.

According to the theory of Varma and Weber the dymamical matrix can be decomposed into $D_{0}+D_{2}$, where $D_{0}$ contains all short range interaction terms (including the first order perturbation term) which can be well described by a Born-von Kármán model. $D_{2}$ arises from the second order perturbation term

$$
D_{2}\left(\kappa \alpha, \kappa^{\prime} \beta \mid \vec{q}\right)=-\sum_{\vec{k}, \mu, \nu} \frac{f_{\vec{k}^{\prime} \nu}-f_{\vec{k} \mu}}{\epsilon_{\vec{k} \mu}-\epsilon_{\vec{k}^{\prime} \nu}} g_{\vec{k} \mu, \vec{k}^{\prime} \nu}^{\kappa \alpha} g_{\vec{k}^{\prime} \nu, \vec{k} \mu}^{\kappa^{\prime} \beta} .
$$

It consists of the electron-phonon interaction parameter $g_{\vec{k}^{\prime} \nu, \vec{k} \mu}^{\kappa^{\prime} \beta}$ which describes the change in the electronic energies by displacing an atom from its equilibrium position. It also contains the single particle energies and the Fermi distribution function to guarentee that occupied states can only couple to unoccupied ones.

It is known from total energy ASW calculations that the ground state of $\alpha$-iron and $\mathrm{Fe}_{3} \mathrm{Ni}$ is ferromagnetic; this is shown in Fig. 2. It should be noted that the use of Eq. 1 is not a good solution because of the energy difference between majority- and minority-spin bands. The difficulty arises from the fact that in second order perturbation theory the phonons do not couple charges belonging to bands with different spin indices. Working on ferromagnetic systems like $\alpha$-iron or $\mathrm{Fe}_{3} \mathrm{Ni}$ one needs 
a more sophisticated ansatz. The easiest model which takes care of spin polarisation would have been a Stoner model with rigid band shifts between spin-up and spin-down bands. Here we have chosen a better procedure by treating minority- and majority-spin energies in two different calculations and then summing up the resulting dynamical matrices $D_{2}$. This leads to

$$
D=D_{0}+D_{2 \uparrow}+D_{2 \downarrow}
$$
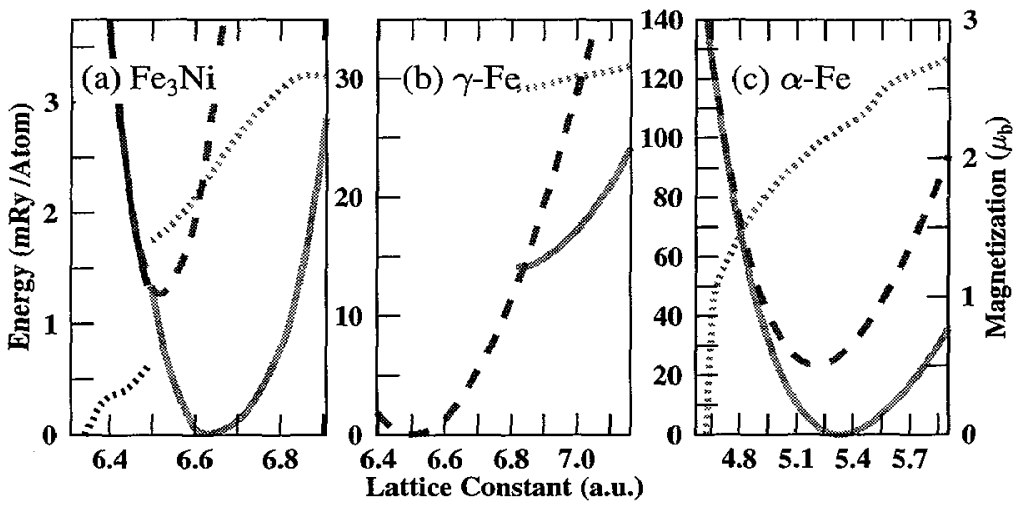

Figure 2: ASW total energies of fcc $\mathrm{Fe}_{3} \mathrm{Ni}$ and fcc/bcc iron. Full lines characterize the ferromagnetic and dashed lines the nonmagnetic state. Dotted curves mark the iron magnetizations per atom. Note that for the case of $\mathrm{Fe}_{3} \mathrm{Ni}$ a low-spin state exists besides the highspin state.

\section{Results}

\subsection{Iron-nickel system}

Inelastic neutron scattering experiments on $\mathrm{Fe}_{65} \mathrm{Ni}_{35}$ were performed by Endoh and Noda [1]. They found an anomalous soft phonon $\mathrm{TA}_{1}$ branch in [110] direction. By fitting their results to a forth order Born-von Kármán model, they were able to reproduce the experimental data quite well. But this force constant model allows no interpretation of the origin of the softening.

Therefore, we have performed calculations for $\mathrm{Fe}_{3} \mathrm{Ni}$ with the first principles theory described in Section 2. The Born-von Kármán model with axial symmetry has been used to get the contribution from the short-range forces including only nearest neighbour and next nearest neighbour iron atoms. In Fig. 3 the acoustic phonon branches of $\mathrm{Fe}_{3} \mathrm{Ni}$ in [110] direction are discussed. As can be seen the

Table 1: Elastic Constants

The elastic constants for $\mathrm{Fe}_{3} \mathrm{Ni}$ compared with experimental results for $\mathrm{Fe}_{65} \mathrm{Ni}_{35}$ taken from [8]. The data are given in $\mathrm{Nm}^{-2}$.

\begin{tabular}{|l|c|c|c|}
\hline \hline Elastic Constants & Experimental & Nonmagnetic $D_{2}$ & Magnetic $D_{2}$ \\
\hline $\mathrm{C}^{\prime}=\left(c_{11}-c_{12}\right) / 2$ & $1.67 * 10^{10}$ & $1.70 * 10^{10}$ & $1.68 * 10^{10}$ \\
\hline $\mathrm{C}_{44}$ & $10.12 * 10^{10}$ & $9.46 * 10^{10}$ & $10.71 * 10^{10}$ \\
\hline$C_{L}=\left(c_{11}+c_{12}+2 c_{44}\right) / 2$ & $24.31 * 10^{10}(\mathrm{US})$ & $22.95 * 10^{10}$ & $25.24 * 10^{10}$ \\
& $30.50 * 10^{10}(\mathrm{INS})$ & & \\
\hline
\end{tabular}

nonmagnetic $D_{2}$ leads to a strong softening especially for $\xi \rightarrow 1.0$, whereas the elastic constants do 


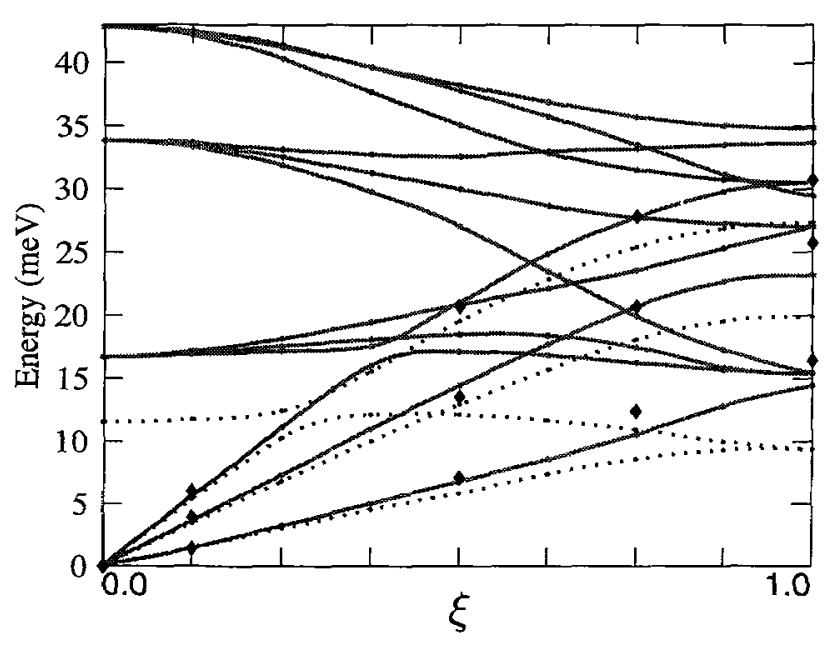

Figure 3: Renormalized acoustic phonon branches of $\mathrm{Fe}_{3} \mathrm{Ni}$ in [110] direction. Lines are a third order polymomial fit through the calculated points. Full lines mark the magnetic case, dashed lines present the nonmagnetic result. Filled diamonds are experimental results [1].

not show any dramatic changes and differ only a bit from the experimental data [8], see for example Table 3.1.

It is obvious from Fig. 3 that with our method to incorperate magnetism we obtain better agreement with experimental results and the elastic constants in Table 3.1. are in excellent agreement with the data from ultrasonic (US) and inelastic neutron scattering (INS) experiments.

The most important fact is that the $\mathrm{TA}_{1}$ mode in $\Gamma$-M dircction has a positive curvature which agrees with the experimental findings. For $\xi$ smaller than 0.5 the experimental values are well reproduced; this good agreement is also reflected by the elastic constants.

For larger phonon wave vectors, especially for $\xi \rightarrow 1.0$, the calculated dispersion curves deviate from the experimental values. This is partially connected with the fact that our calculations have been carried out for stoichiometric $\mathrm{Fe}_{3} \mathrm{Ni}$ yielding acoustic as well as optic phonon branches while the actual samples are disordered. Due to this disorder and due to the fact that iron and nickel have nearly equal masses, neutron spectroscopy gives only acoustic branches. This makes a Born-von Kármán fit difficult.

\subsection{Iron}

Iron is a particularly interesting metal, because it shows martensitic phase transitions as well as magnetovolume instabilities, which leads to Antiinvar behaviour in the fcc structure [9].

In order to analyze iron we have calculated the renormalization of the dynamical matrix for both, $\alpha$ and $\gamma$ phases. The Hamiltonian matrices were determined with the help of ASW calculations as mentioned in Section 2. The $\alpha$-iron ground state is ferromagnetic which is shown in Fig. 2c. The energy of the ferromagnetic ground state is about $22 \mathrm{mRy}$ lower in energy than the nonmagnetic solution. On the other hand the fcc iron can be treated as a nonmagnetic metal.

Employing the theory of Varma and Weber for both systems, we have obtained the perturbation terms $D_{2}$ and phonon dispersions of $\alpha$ - and $\gamma$-iron which are displayed in Fig. 4 and 5. Fig. 4 shows the small softening of the $\mathrm{TA}_{1}[110]$ mode. Our calculations reproduce well the experimental data. Now we have done the same calculations for the low-temperature bcc phase which does not show any softening effects. This agrees also with experimental findings (see Fig. 5). 


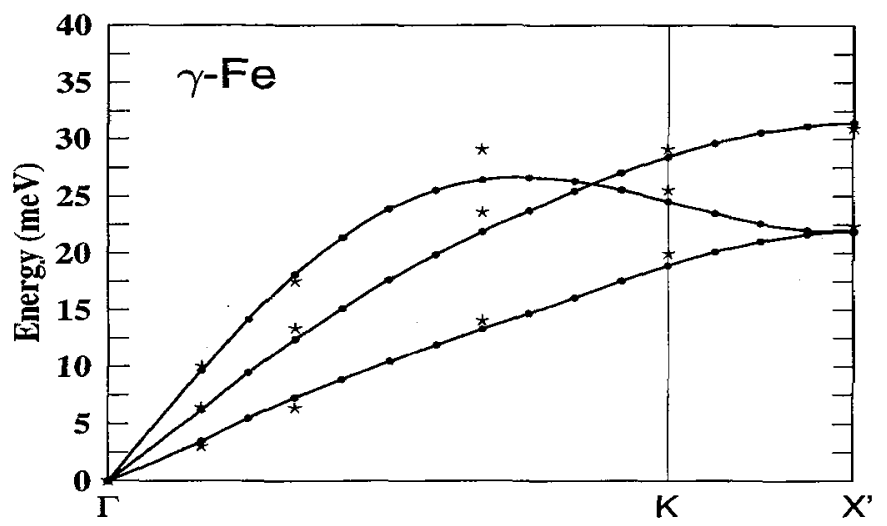

Figure 4: High-temperature phonon dispersion of $\gamma$-Fe in [110] direction. Solid lines are calculated with a nonmagnetic contribution $D_{2}$ (stars). The measurement was done at $1428 \mathrm{~K}$.

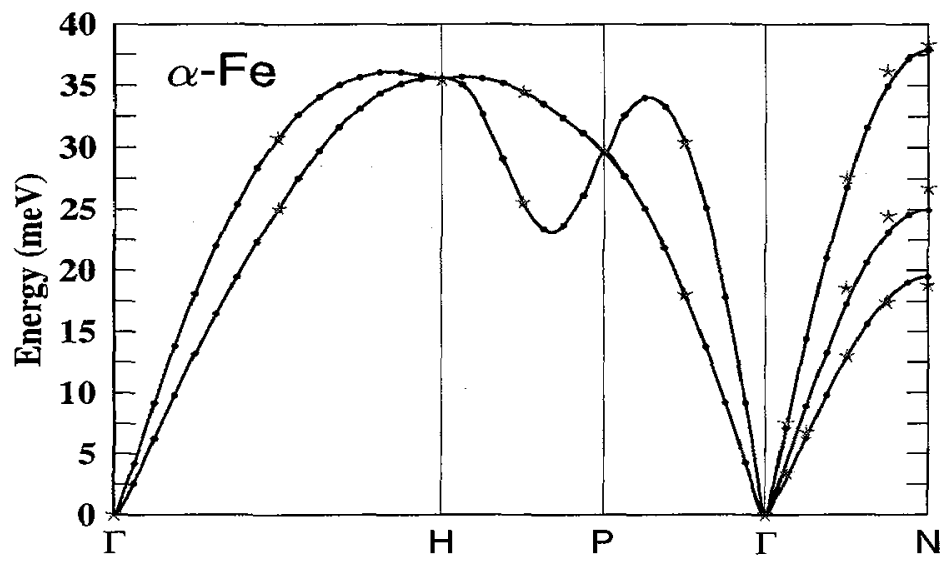

Figure 5: Room-temperature phonon spectrum of $\alpha$-Fe. Dots are calculated points with a magnetic contribution $D_{2}$. The experimental data (stars) were measured at $296 \mathrm{~K}$ [10].

\section{Conclusions}

In the present work we have discussed a few aspects of the connections between the martensitic phase transformation and the Invar effect in iron systems. Our first principles calculations confirmed the experimental findings quite well, except some side effects arising from the differences between our stoichiometric model system and the real material which is disordered.

Furthermore we can conclude that the calculation was improved by taking into account the ferromagnetic character of $\mathrm{Fe}_{3} \mathrm{Ni}$ and $\alpha$-iron. Altogether one can say that the premartensitic softening in Invar alloys occurs for $\xi \rightarrow 0$ and it is a small effect. This is in contrast to large softening effects in nonmagnetic alloys before the actual transformation occurs.

In order to explain the influence of magnetism in Invar-like systems on phonon softening, one has to discuss the electronic density of states (DOS) which is shown in Fig. 6. It can be seen that the spin-polarized density of states near $E_{F}$ is decreased compared to the nonmagnetic case; especially the majority-spin DOS is nearly completely occupied so that the remaining $D_{2}$ comes from the minority-spin bands. Our calculations show that the softening of the $\mathrm{TA}_{1}[110]$ mode in the Invar system $\mathrm{Fe}_{3} \mathrm{Ni}$ is caused by the coupling of phonons to electrons and it is not a pure anharmonic effect caused by phonon-phonon interaction. 


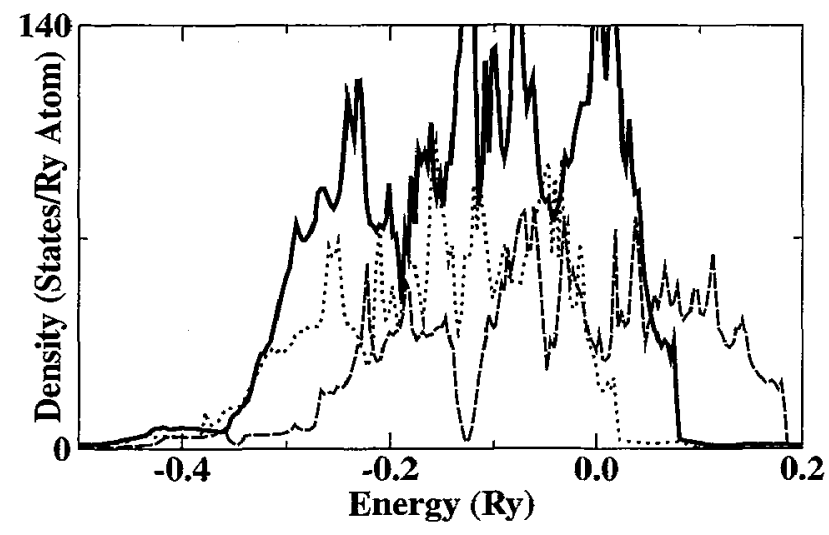

Figure 6: $\mathrm{Fe}_{3} \mathrm{Ni}$ total density of states per unit cell. Full lines mark the nonmagnetic case. The broken lines mark the magnetic DOS (dotted line is the majority and dashed line the minority contribution). The DOS near $\mathrm{E}_{\mathrm{F}}$ (at $0 \mathrm{Ry}$ ) decreases by switching on magnetism.

We find it very interesting that besides Invar also Antiinvar alloys display phonon softening of the same type, namely weak and occuring for $\xi \rightarrow 0$. This was demonstrated by doing calculations for fcc iron exhibiting an anomalously large thermal expansion in its paramagnetic state (this is called Antiinvar bahaviour). If we cool down the system, a martensitic transformation to the $\alpha$ phase occurs. In this phase phonon softening has completely disappeared. Further systematic investigations on other Invar and Antiinvar alloys are needed in order to get a better understanding of the connection between premartensitic behaviour and magnetovolume instabilities.

\section{Acknowledgements}

We would like to thank Prof. Dr. E.F. Wassermann and his collaborators for helpful discussions.

\section{References}

[1] Endoh Y., Noda Y., Ishikawa Y., Solid State Commun. 23 (1977) 951-953.

[2] Zarestky J., and Stassis C., Phys. Rev. B 35 (1987) 4500-4502.

[3] Varma C.M., Weber W., Phys Rev. Lett. 39 (1977) 1094-1098.

[4] Weber W., The Electronic Structure of Complex Systems (NATO ASI Series B, Physics Vol. 113, Plenum, New York, 1984), pp. 345.

[5] Williams A.R., Kübler J., and Gelatt, Jr. C.D., Phys Rev. B 19 (1979) 6094-6018.

[6] Moruzzi V.L., Solid. State Commun. 83 (1992) 739-743.

[7] Entel P., Hoffmann E., Mohn P., Schwarz K., and Moruzzi V.L., Phys. Rev. B 47 (1993) 87068720.

[8] Wassermann E.F., Ferromagnetic Materials (North-Holland, Amsterdam, 1990) pp. 240.

[9] Acet M., Zähres H., Wassermann E.F., and Pepperhoff W., Phys. Rev. B 49 (1994), pp. 6012.

[10] Van Dijk C., and Bergsma J., Neutron Inelastic Scattering (IAEA Vienna 1968, Vol. 1) pp. 233. 